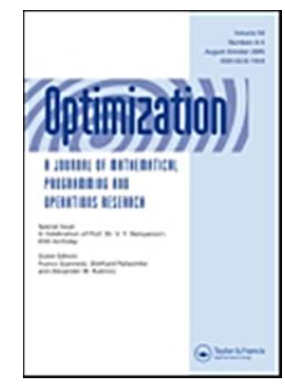

\title{
THE CONTRIBUTION OF K.-H. ELSTER TO GENERALIZED CONJUGATION THEORY AND NONCONVEX DUALITY
}

\begin{tabular}{|r|l|}
\hline Journal: & Optimization \\
\hline Manuscript ID: & GOPT-2014-0080.R1 \\
\hline Manuscript Type: & Original Article \\
\hline Date Submitted by the Author: & 28-Apr-2014 \\
\hline Complete List of Authors: & Martinez-Legaz, Juan Enrique; Universitat Autonoma de Barcelona, ; \\
\hline Keywords: & $\begin{array}{l}\text { Generalized conjugation, nonconvex optimization, duality theory, } \\
\text { generalized convexity, optimality conditions }\end{array}$ \\
\hline \multicolumn{2}{|l}{} \\
\hline
\end{tabular}

\section{SCHOLARONE ${ }^{\text {m }}$}

Manuscripts 
This is a Submitted Manuscript of an article published by Taylor \& Francis in Optimization, on 2015, Vol.64, Iss. 1, available online: • https://doi.org/10.1080/02331934.2014.929683

\title{
THE CONTRIBUTION OF K.-H. ELSTER TO GENERALIZED CONJUGATION THEORY AND NONCONVEX DUALITY*
}

\author{
Juan Enrique Martínez-Legaz \\ Departament d'Economia i d'Història Econòmica \\ Universitat Autònoma de Barcelona \\ 08193 Bellaterra, Spain
}

\begin{abstract}
This article surveys the main contributions of K.-H. Elster to the theory of generalized conjugate functions and its applications to duality in nonconvex optimization.
\end{abstract}

Keywords: Generalized conjugation, nonconvex optimization, duality theory, generalized convexity, optimality conditions, fractional programming, geometric vector inequality

Mathematics Subject Classification 1991: Primary: 90C26, 49N15, 52A01; Secondary: 90C32, 90C29, 90A14

\section{Introduction}

Since the seminal work of W. Fenchel [18], the theory of conjugate functions plays a fundamental role in convex optimization, especially in connection with duality theory. With the main objective of extending convex duality theory to the

*The author was supported by the MICINN of Spain, Grant MTM2011-29064-C03-01, and under Australian Research Council's Discovery Projects funding scheme (project number DP140103213). He is affiliated to MOVE (Markets, Organizations and Votes in Economics). 
nonconvex case, many generalized conjugation concepts have been introduced in the literature (see [21] and the references contained therein). One of the main researchers in this field was K.-H. Elster, who already more than twenty years ago proposed in a joint paper with R. Deumlich [1] one of the pioneering generalized conjugation notions, called $\Phi$-conjugation, based on geometrical properties of Fenchel conjugation. Between 1977 and 1990 he published several papers devoted to this notion as well as to other generalized conjugation concepts, and developed their application to duality theory in nonconvex optimization. The aim of this article is to survey the main ideas and results presented in those papers.

Let us recall some basic concepts and notation that will be used throughout the paper. Even though most results are valid in an infinite-dimensional setting (see, e.g., [8]), for simplicity we shall only consider finite-dimensional spaces. The domain, the graph, the epigraph and the hypograph of $f: \mathbb{R}^{n} \rightarrow \mathbb{R} \cup\{ \pm \infty\}$ are the sets dom $f=\left\{x \in \mathbb{R}^{n} / f(x) \in \mathbb{R}\right\}$, graph $f=\left\{(x, z) \in \mathbb{R}^{n} \times \mathbb{R} / f(x)=z\right\}$, epi $f=\left\{(x, z) \in \mathbb{R}^{n} \times \mathbb{R} / f(x) \leq z\right\}$ and hypo $f=\left\{(x, z) \in \mathbb{R}^{n} \times \mathbb{R} / f(x) \geq z\right\}$, respectively. A vector $x^{*} \in \mathbb{R}^{n}$ is said to be a subgradient of $f$ at $x_{0} \in \operatorname{dom} f$ if

$$
f(x) \geq f\left(x_{0}\right)+x^{* T}\left(x-x_{0}\right) \quad \forall x \in \operatorname{dom} f
$$

(vectors in $\mathbb{R}^{n}$ are to be interpreted as column vectors and the superscript ${ }^{T}$ denotes transpose). The set $\partial f\left(x_{0}\right)$ of all subgradients of $f$ at $x_{0}$ is called the subdifferential of $f$ at $x_{0}$. The superdifferential of $f$ at $x_{0}$ is the set $\partial_{u} f\left(x_{0}\right)=$ $-\partial(-f)\left(x_{0}\right)$; it consists of all supergradients of $f$ at $x_{0}$, that is, all vectors $x^{*} \in \mathbb{R}^{n}$ such that

$$
f(x) \leq f\left(x_{0}\right)+x^{* T}\left(x-x_{0}\right) \quad \forall x \in \operatorname{dom} f .
$$

The Euclidean norm will be denoted by $\|$.$\| . For a set C \subseteq \mathbb{R}^{n}$, ri $C$ and $c l C$ will mean the relative interior and the closure of $C$, respectively.

\section{2. $\Phi$-conjugate functions $[1,11,12,3,4]$}

The main notion of generalized conjugate function due to K.-H. Elster and his collaborators is that of $\Phi$-conjugation. It is based on an interpretation of the convex conjugation operator in terms of polarity with respect to a paraboloid [18]. Recall that the polar of a point $x_{0} \in \mathbb{R}^{n}$ with respect to the regular quadric hypersurface $\left(\begin{array}{ll}1 & x^{T}\end{array}\right)\left(\begin{array}{ll}a_{0} & a^{T} \\ a & C\end{array}\right)\left(\begin{array}{l}1 \\ x\end{array}\right)=0$, where $C$ is a symmetric $n x n$ 
matrix, $a \in \mathbb{R}^{n}, a_{0} \in \mathbb{R}$, and $\operatorname{det}\left(\begin{array}{cc}a_{0} & a^{T} \\ a & C\end{array}\right) \neq 0$, is the hyperplane of equation

$$
\left(\begin{array}{ll}
1 & x_{0}^{T}
\end{array}\right)\left(\begin{array}{ll}
a_{0} & a^{T} \\
a & C
\end{array}\right)\left(\begin{array}{l}
1 \\
x
\end{array}\right)=0 .
$$

Let $f: G \rightarrow \mathbb{R}$ be a convex function defined on the convex region $G \subseteq \mathbb{R}^{n}$, and consider the paraboloid in $\mathbb{R}^{n+1}$ consisting of all points $\left(x_{1}, \ldots, x_{n}, x_{n+1}\right)$ satisfying

$$
x_{1}^{2}+\ldots+x_{n}^{2}-2 x_{n+1}=0 .
$$

The envelope of the polar hyperplanes of the points of the hypersurface $x_{n+1}=$ $f\left(x_{1}, \ldots, x_{n}\right)$ is the hypersurface $x_{n+1}^{*}=f^{*}\left(x_{1}^{*}, \ldots, x_{n}^{*}\right)$, that is, the graph of the Fenchel conjugate $f^{*}$ of $f$ defined by $f^{*}\left(x_{1}^{*}, \ldots, x_{n}^{*}\right)=\sup \left\{\sum_{i=1}^{n} x_{i} x_{i}^{*}-f\left(x_{1}, \ldots, x_{n}\right)\right\}$ on the set $G^{*}$ of points $\left(x_{1}^{*}, \ldots, x_{n}^{*}\right)$ for which this supremum is finite. More explicitely, $x_{n+1}^{*}=f^{*}\left(x_{1}^{*}, \ldots, x_{n}^{*}\right)$ is the envelope of the hyperplanes $x_{1} x_{1}^{*}+\ldots+$ $x_{n} x_{n}^{*}-x_{n+1}^{*}=x_{n+1}$ corresponding to the points $\left(x_{1}, \ldots, x_{n}, x_{n+1}\right)$ of the hypersurface $x_{n+1}=f\left(x_{1}, \ldots, x_{n}\right)$.

The notion of $\Phi$-conjugation is based on replacing the hyperparaboloid (2.1) by an arbitrary regular quadric hypersurface $\Phi$ in $\mathbb{R}^{n+1}$, defined by the equation

$$
\left(\begin{array}{lll}
1 & x^{T} & x_{n+1}
\end{array}\right) A\left(\begin{array}{c}
1 \\
x \\
x_{n+1}
\end{array}\right)=0, \text { with } A=\left(\begin{array}{ccc}
a_{0} & a^{T} & c \\
a & B & e \\
c & e^{T} & b
\end{array}\right)
$$

where $\left(x, x_{n+1}\right) \in \mathbb{R}^{n} \times \mathbb{R},\left(a_{0}, a, c\right) \in \mathbb{R} \times \mathbb{R}^{n} \times \mathbb{R}, B$ is a symmetric $n \times n$ matrix, $(e, b) \in \mathbb{R}^{n} \times \mathbb{R}$, and the matrix $A$ is assumed to be nonsingular. The conjugates of a set are defined as follows:

Definition 1. Let $F \subseteq \mathbf{R}^{n+1}$ be a nonempty set, $\Phi$ be the regular quadric hypersurface in $\mathbb{R}^{n+1}$ defined by (2.2), and $h: \mathbb{R}^{n} \times \mathbb{R} \times \mathbb{R}^{n} \times \mathbb{R} \rightarrow \mathbb{R}$ be the function defined by $h\left(x, z, x^{*}, z^{*}\right)=\left(\begin{array}{lll}1 & x^{T} & z\end{array}\right) A\left(\begin{array}{c}1 \\ x^{*} \\ z^{*}\end{array}\right)$. Then the sets

$$
F_{\Phi}=\left\{\left(x^{*}, z^{*}\right) / \inf _{(x, z) \in F} h\left(x, z, x^{*}, z^{*}\right)=0\right\}
$$

and

$$
F^{\Phi}=\left\{\left(x^{*}, z^{*}\right) / \sup _{(x, z) \in F} h\left(x, z, x^{*}, z^{*}\right)=0\right\}
$$


are called the lower $\Phi$-conjugate of $F$ and the upper $\Phi$-conjugate of $F$, respectively.

The corresonding definitions for functions are given next:

Definition 2. Let $f: \mathbb{R}^{n} \rightarrow \mathbb{R} \cup\{ \pm \infty\}$ be such that

$$
\text { graph } f \subseteq\left\{(x, z) / c+e^{T} x+b z \neq 0\right\},
$$

and set

$$
k\left(x, z, x^{*}\right)=-\frac{h\left(x, z, x^{*}, 0\right)}{c+e^{T} x+b z} .
$$

Then the extended real valued functions $f_{\Phi}$ and $f^{\Phi}$ defined by

$$
f_{\Phi}\left(x^{*}\right)=\inf _{x \in \operatorname{dom} f} k\left(x, f(x), x^{*}\right)
$$

and

$$
f^{\Phi}\left(x^{*}\right)=\sup _{x \in \operatorname{dom} f} k\left(x, f(x), x^{*}\right)
$$

are called the lower $\Phi$-conjugate function of $f$ and the upper $\Phi$-conjugate function of $f$, respectively.

Some fundamental properties of $\Phi$-conjugate functions are collected in the following theorem, the proof of which can found in [1].

Theorem 1. [1, 12, 4] For any function $f: \mathbb{R}^{n} \rightarrow \mathbb{R} \cup\{ \pm \infty\}$ the following statements hold:

(i) $f_{\Phi}$ is concave and upper semicontinuous.

(ii) $f^{\Phi}$ is convex and lower semicontinuous.

(iii) If graph $f \subseteq\left\{(x, z) / c+e^{T} x+b z>0\right\}$ then the following inequalities are satisfied:

$$
\begin{array}{ll}
h\left(x, f(x), x^{*}, f^{\Phi}\left(x^{*}\right)\right) \geq 0 & \forall\left(x, x^{*}\right) \in \operatorname{dom} f \times \operatorname{dom} f^{\Phi}, \\
h\left(x, f(x), x^{*}, f_{\Phi}\left(x^{*}\right)\right) \leq 0 & \forall\left(x, x^{*}\right) \in \operatorname{dom} f \times \operatorname{dom} f_{\Phi} ;
\end{array}
$$

if, instead, graph $f \subseteq\left\{(x, z) / c+e^{T} x+b z<0\right\}$, then the opposite inequalities are satisfied.

Second $\Phi$-conjugate functions are also of capital importance; they are defined as follows: 
Definition 3. Let $f: \mathbb{R}^{n} \rightarrow \mathbb{R} \cup\{ \pm \infty\}$. If $b \geq 0$ and the graphs of $f$ and $f^{\Phi}$ are contained in the open halfspace $\left\{\left(x^{*}, z\right) / c+e^{T} x^{*}+b z>0\right\}$ then the function $f^{\Phi \Phi}=\left(f^{\Phi}\right)^{\Phi}$ is said to be the upper $\Phi$-biconjugate function of $f$, and the function $f_{\Phi \Phi}$ defined by

$$
f_{\Phi \Phi}\left(x^{*}\right)=\inf _{c+e^{T} x+b f_{\Phi}\left(x^{*}\right)>0} k\left(x^{*}, f_{\Phi}\left(x^{*}\right), x\right)
$$

is said to be the lower $\Phi$-biconjugate function of $f$.

If $b<0$ and the graphs of $f$ and $f_{\Phi}$ are contained in the open halfspace $\left\{\left(x^{*}, z\right) / c+e^{T} x^{*}+b z>0\right\}$ then the function $f_{\Phi \Phi}=\left(f_{\Phi}\right)_{\Phi}$ is said to be the lower $\Phi$-biconjugate function of $f$, and the function $f^{\Phi \Phi}$ defined by

$$
f^{\Phi \Phi}\left(x^{*}\right)=\sup _{c+e^{T} x+b f^{\Phi}\left(x^{*}\right)>0} k\left(x^{*}, f^{\Phi}\left(x^{*}\right), x\right)
$$

is said to be the upper $\Phi$-biconjugate function of $f$.

The relations between an arbitrary function and its $\Phi$-biconjugate functions are given next:

Theorem 2. [4] If the graph of $f: \mathbb{R}^{n} \rightarrow \mathbb{R} \cup\{ \pm \infty\}$ is contained in the open halfspace $\left\{(x, z) / c+e^{T} x+b z>0\right\}$ then the following statements hold:

(i) If either $b \geq 0$ and graph $f^{\Phi} \subseteq\left\{\left(x^{*}, z\right) / c+e^{T} x^{*}+b z>0\right\}$ or $b<0$ and graph $f_{\Phi} \subseteq\left\{\left(x^{*}, z\right) / c+e^{T} x^{*}+b z>0\right\}$ then

$$
f^{\Phi \Phi}(x) \leq f(x) \leq f_{\Phi \Phi}(x) \quad \forall x \in \text { dom } f .
$$

(ii) If $b \geq 0$ and the graph of $f^{\Phi}\left(\right.$ of $\left.f_{\Phi}\right)$ is contained in the open halfspace $\left\{\left(x^{*}, z\right) / c+e^{T} x^{*}+b z>0\right\}$ and there is a supporting hyperplane $z=m^{T} x+\beta$ of epi $f$ (resp., hypo $f$ ) at $\left(x_{0}, f\left(x_{0}\right)\right.$ ) such that for $\left(\begin{array}{c}\widehat{t} \\ \widehat{x} \\ \widehat{z}\end{array}\right)=A^{-1}\left(\begin{array}{l}1 \\ 0 \\ 0\end{array}\right)$ one has $\widehat{z}-m^{T} \widehat{x}-\beta \widehat{t}>0$ then $f^{\Phi \Phi}\left(x_{0}\right)=f\left(x_{0}\right)$ (resp., $f_{\Phi \Phi}\left(x_{0}\right)=f\left(x_{0}\right)$ ).

The assumption in statement (ii) of the preceding theorem about the existence of a supporting hyperplane to the epigraph (hypograph) of $f$ at $\left(x_{0}, f\left(x_{0}\right)\right)$ is of course related to the nonemptiness of the subdifferential (resp., the superdifferential) of $f$ at $x_{0}$; namely, it is well known in convex analysis that the hyperplane $z=m^{T} x+\beta$ is a supportimg hyperplane of epi $f($ hypo $f)$ at $\left(x_{0}, f\left(x_{0}\right)\right)$ if and only if $m \in \partial f\left(x_{0}\right)$ (resp., $\left.m \in-\partial(-f)\left(x_{0}\right)\right)$ and $\beta=f\left(x_{0}\right)-m^{T} x_{0}$. 
An abstract approach to $\Phi$-conjugation theory is provided in $[15,16]$, where a notion of conjugation associated to a polarity between two sets $X$ and $Y$ (that is, a mapping from $2^{X}$ into $2^{Y}$ which transforms unions into intersections) is introduced. This notion allows for unifying $\Phi$-conjugation and the generalized Fenchel conjugation theory introduced by Moreau [20]. Some other results on this concept of conjugation are provided in [14].

\section{Applications of $\Phi$-conjugation to nonconvex optimization $[2,12,4,5,6,7,8]$}

The most typical application of generalized conjugation is to duality theory in optimization. In the case of $\Phi$-conjugate functions, assuming that the matrix $A$ of (2.2) satisfies $c^{2}+\|e\|^{2}+b^{2}>0$ and that the functions $f: \mathbb{R}^{n} \rightarrow \mathbb{R} \cup\{ \pm \infty\}$ and $g: \mathbb{R}^{n} \rightarrow \mathbb{R} \cup\{ \pm \infty\}$ are such that graph $f^{\Phi} \subseteq\left\{\left(x^{*}, z\right) / c+e^{T} x^{*}+b z \neq 0\right\}$ and graph $g \subseteq\left\{(x, z) / c+e^{T} x+b z>0\right\}$, one considers the pair of dual problems

$\left(P_{1}\right) \quad$ Minimize $p_{1}(x)=\frac{c+e^{T} x+b f(x)}{c+e^{T} x+b g(x)}, \quad x \in \operatorname{dom} f \cap \operatorname{dom} g$,

$\left(D_{1}\right) \quad$ Maximize $d_{1}\left(x^{*}\right)=\frac{c+e^{T} x^{*}+b g_{\Phi}\left(x^{*}\right)}{c+e^{T} x^{*}+b f^{\Phi}\left(x^{*}\right)}, \quad x^{*} \in$ dom $f^{\Phi} \cap \operatorname{dom} g_{\Phi}$, in the case $b \neq 0$, and

$\left(P_{2}\right) \quad$ Minimize $p_{2}(x)=\frac{f(x)-g(x)}{c+e^{T} x}, \quad x \in \operatorname{dom} f \cap \operatorname{dom} g$,

$\left(D_{2}\right) \quad$ Maximize $d_{2}\left(x^{*}\right)=\frac{g_{\Phi}\left(x^{*}\right)-f^{\Phi}\left(x^{*}\right)}{c+e^{T} x^{*}}, \quad x \in \operatorname{dom} f^{\Phi} \cap \operatorname{dom} g_{\Phi}$, in the case $b=0$. The following weak duality theorem holds:

Theorem 1. [4] Let the graphs of $f, g$ and $f^{\Phi}$ be contained in the open halfspace $\left\{(x, z) / c+e^{T} x+b z>0\right\}$ and $b \geq 0$. Then

$$
\inf _{x \in \operatorname{dom} f \cap d o m} p_{j}(x) \geq \sup _{x^{*} \in \operatorname{dom}} f_{f^{\Phi} \cap \operatorname{dom}} d_{g_{\Phi}}\left(x^{*}\right) \quad(j=1,2) .
$$

Corollary 2. [4] Under the assumptions of Theorem 1, if $j \in\{1,2\}, x_{0} \in$ dom $f \cap \operatorname{dom} g$ and $x_{0}^{*} \in \operatorname{dom} f^{\Phi} \cap \operatorname{dom} g_{\Phi}$ are such that $p_{j}\left(x_{0}\right)=d_{j}\left(x_{0}^{*}\right)$ then $x_{0}$ and $x_{0}^{*}$ are optimal solutions of $\left(P_{j}\right)$ and $\left(D_{j}\right)$, respectively.

To get strong duality theorems, some suitable convexity assumptions are required. As in the previous section, we denote $\left(\begin{array}{c}\widehat{t} \\ \widehat{x} \\ \widehat{z}\end{array}\right)=A^{-1}\left(\begin{array}{l}1 \\ 0 \\ 0\end{array}\right)$. 
Theorem 3. [2, 4] Assume that $f$ is convex, $g$ is concave and the hypotheses of Theorem 1 hold. If $b>0$ and the conditions

(i) ri dom $f \cap$ ri dom $g \neq \emptyset$,

(ii) $\widehat{t}<0$,

(iii) $\frac{\widehat{x}}{\widehat{t}} \in \operatorname{cl}(\operatorname{dom} f \cap \operatorname{dom} g)$,

(iv) $\varlimsup_{\substack{\frac{\hat{x}}{t}+\delta \in \operatorname{dom} \\ g}} g\left(\frac{\widehat{x}}{\widehat{t}}+\delta\right)>\frac{\widehat{z}}{\widehat{t}}$,

(v) $\inf _{x \in \text { dom }}$ fndom $g\left\{c+e^{T} x+b f(x)\right\}>0$

are satisfied then there exists $x_{0}^{*} \in \operatorname{dom} f^{\Phi} \cap$ dom $g_{\Phi}$ such that

$$
\inf _{x \in \text { dom fกdom }} p_{1}(x)=\max _{x^{*} \in \text { dom } f^{\Phi} \cap \text { dom }} d_{\Phi} d_{1}\left(x^{*}\right)=d_{1}\left(x_{0}^{*}\right) .
$$

Theorem 4. $[2,4,7]$ Assume that $f$ is convex, $g$ is concave and the hypotheses of Theorem 1 hold. If $b>0$ and the conditions

(i) ri dom $f \cap$ ri dom $g \neq \emptyset$,

(ii) $\widehat{t} \leq 0$,

(iii) $\frac{\widehat{x}}{\widehat{t}} \in \operatorname{cl}(\operatorname{dom} f \cap \operatorname{dom} g)$, if $\widehat{t}<0$,

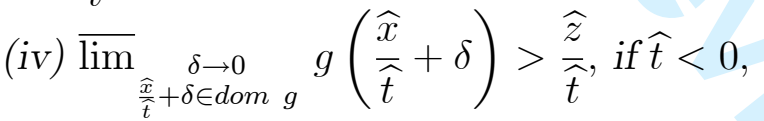

(v) $\inf _{x \in \text { dom } f \cap \operatorname{dom} g} p_{2}(x)>-\infty$

are satisfied then there exists $x_{0}^{*} \in \operatorname{dom} f^{\Phi} \cap \operatorname{dom} g_{\Phi}$ such that

$$
\inf _{x \in \text { dom fndom } g} p_{2}(x)=\max _{x^{*} \in \text { dom } f^{\Phi} \cap \text { dom }} d_{g_{\Phi}} d_{2}\left(x^{*}\right)=d_{2}\left(x_{0}^{*}\right) .
$$

The following theorem gives optimality conditions for problems $\left(P_{j}\right)$ and $\left(D_{j}\right)$ $(j=1,2)$ in geometrical terms:

Theorem 5. [2, 4] Let the graphs of $f, g$ and $f^{\Phi}$ be contained in the open halfspace $\left\{(x, z) / c+e^{T} x+b z>0\right\}, b \geq 0, x_{0} \in \operatorname{dom} f \cap \operatorname{dom} g, x_{0}^{*} \in$ dom $f^{\Phi} \cap$ dom $g_{\Phi}$ and $j \in\{1,2\}$.

(1) The following statements are equivalent:

(i) $x_{0}$ and $x_{0}^{*}$ are optimal solutions of $\left(P_{j}\right)$ and $\left(D_{j}\right)$, respectively, and

$$
p_{j}\left(x_{0}\right)=d_{j}\left(x_{0}^{*}\right) .
$$


(ii) There exist $\alpha_{1}^{*}, \alpha_{2}^{*} \in \mathbb{R}$ with $\left(x_{0}^{*}, \alpha_{1}^{*}\right),\left(x_{0}^{*}, \alpha_{2}^{*}\right) \in\left\{(x, z) / c+e^{T} x+b z>0\right\}$ such that

$$
z=k\left(x_{0}^{*}, \alpha_{1}^{*}, x\right)
$$

is a supporting hyperplane of epi $f$ at $\left(x_{0}, f\left(x_{0}\right)\right)$ and

$$
z=k\left(x_{0}^{*}, \alpha_{2}^{*}, x\right)
$$

is a supporting hyperplane of hypo $g$ at $\left(x_{0}, g\left(x_{0}\right)\right)$.

(2) If the equality (3.1) holds then there exist $\alpha_{1}, \alpha_{2} \in \mathbb{R}$ with $\left(x_{0}, \alpha_{1}\right),\left(x_{0}, \alpha_{2}\right) \in$ $\left\{(x, z) / c+e^{T} x+b z>0\right\}$ such that

$$
z^{*}=k\left(x_{0}, \alpha_{1}, x^{*}\right)
$$

is a supporting hyperplane of epi $f^{\Phi}$ at $\left(x_{0}^{*}, f^{\Phi}\left(x_{0}^{*}\right)\right)$ and

$$
z^{*}=k\left(x_{0}, \alpha_{2}, x^{*}\right)
$$

is a supporting hyperplane of hypo $g_{\Phi}$ at $\left(x_{0}^{*}, g_{\Phi}\left(x_{0}^{*}\right)\right)$.

(3) If $f$ is convex, $g$ is concave, $\{x / \widehat{t x}=\widehat{x}\} \neq \emptyset$, and there exist $\alpha_{1}, \alpha_{2} \in \mathbb{R}$ with $\left(x_{0}, \alpha_{1}\right),\left(x_{0}, \alpha_{2}\right) \in\left\{(x, z) / c+e^{T} x+b z>0\right\}$ such that (3.2) is a supporting hyperplane of epi $f^{\Phi}$ at $\left(x_{0}^{*}, f^{\Phi}\left(x_{0}^{*}\right)\right)$ and (3.3) is a supporting hyperplane of hypo $g_{\Phi}$ at $\left(x_{0}^{*}, g_{\Phi}\left(x_{0}^{*}\right)\right)$ then $x_{0}^{*}$ is an optimal solution of $\left(D_{j}\right)$ and there exists a sequence $\left\{x_{\nu}\right\}$ in dom $f \cap$ dom $g$ converging to $x_{0}$ such that $\lim _{\nu \rightarrow \infty} p_{j}\left(x_{\nu}\right)=$ $d_{j}\left(x_{0}^{*}\right)$.

By the equivalence between supporting hyperplanes to epigraphs (hypographs) and subdifferentials (resp., superdifferentials) that has been recalled at the end of the preceding section, one can give analytic versions of the preceding theorem $[4$, Thm. 3.7 and Cor. 3.7].

The special case when $c=0, e=0$ and $b=1$, which corresponds to ordinary fractional programming, was anlayzed in more detail in [7].

Example 1. [5] Let $f: \mathbb{R} \rightarrow \mathbb{R}$ and $g:(-\sqrt{5}, \sqrt{5}) \rightarrow \mathbb{R}$ be given by $f(x)=$ $\frac{1}{2}(x+3)^{2}+2$ and $g(x)=-\frac{1}{2} x^{2}+\frac{5}{2}$, respectively, and consider the optimization problem

$$
\text { Minimize } p_{1}(x)=\frac{f(x)}{g(x)}, \quad x \in(-\sqrt{5}, \sqrt{5})
$$


which has $x_{0}=-1$ as its unique optimal solution, with $p_{1}\left(x_{0}\right)=2$. This problem has the format $\left(P_{1}\right)$; to introduce a dual problem with format $\left(D_{1}\right)$, we choose

$$
A=\left(\begin{array}{ccc}
-\rho^{2} & 0 & 0 \\
0 & 1 & 0 \\
0 & 0 & 1
\end{array}\right), \quad \rho>0
$$

Then the $\Phi$-conjugate functions $f^{\Phi}: \mathbb{R} \rightarrow \mathbb{R}$ and $g_{\Phi}:\left[-\frac{\rho^{2}}{\sqrt{5}}, \frac{\rho^{2}}{\sqrt{5}}\right] \rightarrow \mathbb{R}$ are gven by

$$
f^{\Phi}\left(x^{*}\right)=\frac{1}{4}\left(\sqrt{\rho^{4}+6 \rho^{2} x^{*}+13 x^{* 2}}+\rho^{2}+3 x^{*}\right)
$$

and

$$
g_{\Phi}\left(x^{*}\right)=\frac{1}{5}\left(\sqrt{\rho^{4}-5 x^{* 2}}+\rho^{2}\right) ;
$$

therefore, the dual problem $\left(D_{1}\right)$ reduces to

Maximize

$$
d_{1}\left(x^{*}\right)=\frac{\frac{1}{5}\left(\sqrt{\rho^{4}-5 x^{* 2}}+\rho^{2}\right)}{\frac{1}{4}\left(\sqrt{\rho^{4}+6 \rho^{2} x^{*}+13 x^{* 2}}+\rho^{2}+3 x^{*}\right)}, \quad x^{*} \in\left[-\frac{\rho^{2}}{\sqrt{5}}, \frac{\rho^{2}}{\sqrt{5}}\right],
$$

which has $x_{0}^{*}=-\frac{\rho^{2}}{3}$ as its unique optimal solution, with $d_{1}\left(x_{0}^{*}\right)=2=p_{1}\left(x_{0}\right)$. The hyperplanes $z=2 x+6$ and $z=x+3$ support epi $f$ at $\left(x_{0}, f\left(x_{0}\right)\right)$ and hypo $g$ at $\left(x_{0}, g\left(x_{0}\right)\right)$, respectively. Similarly, the hyperplanes $4 z^{*}=x^{*}+\rho^{2}$ and $2 z^{*}=x^{*}+\rho^{2}$ support epi $f^{\Phi}$ at $\left(x_{0}^{*}, f^{\Phi}\left(x_{0}^{*}\right)\right)$ and hypo $g_{\Phi}$ at $\left(x_{0}^{*}, g_{\Phi}\left(x_{0}^{*}\right)\right)$, respectively.

Example 2. [5] Let $a \in \mathbb{R}^{n}$ and $b, c>0$, define $f:\left\{x / a^{T} x+b>0\right\} \rightarrow \mathbb{R}$ and $g:\{x /\|x\|<\sqrt{2 c}\} \rightarrow \mathbb{R}$ by $f(x)=a^{T} x+b$ and $g(x)=-\frac{1}{2}\|x\|^{2}+c$, respectively, and consider the optimization problem

which has

$$
\text { Minimize } p_{1}(x)=\frac{f(x)}{g(x)}, \quad x \in\left\{x / a^{T} x+b>0,\|x\|<\sqrt{2 c}\right\},
$$

$$
x_{0}= \begin{cases}-\frac{a}{\|a\|^{2}}\left(b-\sqrt{b^{2}-2 c\|a\|^{2}}\right), & \text { if } a \neq 0 \\ 0, & \text { if } a=0\end{cases}
$$


as its unique optimal solution. As in the preceding example, this problem has the format $\left(P_{1}\right)$; we choose again the matrix

$$
A=\left(\begin{array}{ccc}
-\rho^{2} & 0 & 0 \\
0 & I_{n} & 0 \\
0 & 0 & 1
\end{array}\right), \quad \rho>0, I_{n} \text { the } n \times n \text { identity matrix. }
$$

Then the $\Phi$-conjugate functions $f^{\Phi}:\left\{-\frac{\rho^{2}}{b}\right\} \rightarrow \mathbb{R}$ and $g_{\Phi}:\left\{y \in \mathbb{R}^{n} /\|y\| \leq \sqrt{2 c}\right\} \rightarrow \mathbb{R}$ are gven by

$$
f^{\Phi}\left(x^{*}\right)=\frac{\rho^{2}}{b}
$$

and

$$
g_{\Phi}\left(x^{*}\right)=\frac{1}{2 c}\left(\rho^{2}+\sqrt{\rho^{4}-2 c\left\|x^{*}\right\|^{2}}\right)
$$

therefore, the dual problem $\left(D_{1}\right)$ reduces to

Maximize

$$
d_{1}\left(x^{*}\right)=\frac{\frac{1}{2 c}\left(\rho^{2}+\sqrt{\rho^{4}-2 c\left\|x^{*}\right\|^{2}}\right)}{\frac{\rho^{2}}{b}}, \quad x^{*} \in\left\{-\frac{\rho^{2}}{b}\right\},
$$

which has $x_{0}^{*}=-\frac{\rho^{2}}{b}$ as its unique feasible (hence, optimal) solution, with $d_{1}\left(x_{0}^{*}\right)=$ $\frac{1}{2 c}\left(b+\sqrt{b^{2}-2 c\|a\|^{2}}\right)$. Therefore, from Theorem 4 it follows that $\inf _{a^{T} x+b>0,\|x\|<\sqrt{2 c}} p_{1}(x)=$ $\frac{1}{2 c}\left(b+\sqrt{b^{2}-2 c\|a\|^{2}}\right)$.

An analytic characterization of dual pairs of optimal solutions in the case when strong duality holds is given in the next theorem:

Theorem 6. [6] If $b \geq 0$ and graph $f \cup$ graph $g \subseteq\left\{(x, z) / c+e^{T} x+b z>0\right\}$ then the following statements hold:

(i) The following assertions are equivalent:

(1) $x_{0} \in \operatorname{dom} f \cap$ dom $g$ and $x_{0}^{*} \in$ dom $f^{\Phi} \cap$ dom $g_{\Phi}$ are solutions of the optimization problems $\left(P_{i}\right)$ and $\left(D_{i}\right)$, respectively and $p_{i}\left(x_{0}\right)=d_{i}\left(x_{0}^{*}\right), I=1,2$. 
that

(2) There exist pairs $\left(x_{0}^{*}, \alpha_{1}^{*}\right),\left(x_{0}^{*}, \alpha_{2}^{*}\right) \in\left\{(x, z) / c+e^{T} x+b z>0\right\}$ such

$$
\begin{aligned}
& \text { (a) } h\left(x_{0}^{*}, \alpha_{1}^{*}, x_{0}, f\left(x_{0}\right)\right)=0, h\left(x_{0}^{*}, \alpha_{2}^{*}, x_{0}, g\left(x_{0}\right)\right)=0 . \\
& \text { (b) }-\frac{a+B x_{0}^{*}+e \alpha_{1}^{*}}{c+e^{T} x_{0}^{*}+b \alpha_{1}^{*}} \in \partial f\left(x_{0}\right),-\frac{a+B x_{0}^{*}+e \alpha_{2}^{*}}{c+e^{T} x_{0}^{*}+b \alpha_{2}^{*}} \in \partial_{u} g\left(x_{0}\right) .
\end{aligned}
$$

(ii) If statement (i)(1) holds, then there exist $\alpha_{j} \in \mathbb{R}, j=1,2$, such that $c+e^{T} x_{0}+b \alpha_{j}>0$ and

(3) $h\left(x_{0}^{*}, f^{\Phi}\left(x_{0}^{*}\right), x_{0}, \alpha_{1}\right)=0, h\left(x_{0}^{*}, g_{\Phi}\left(x_{0}^{*}\right), x_{0}, \alpha_{2}\right)=0$,

$$
\text { (4) }-\frac{a+B x_{0}+e \alpha_{1}}{c+e^{T} x_{0}+b \alpha_{1}} \in \partial f^{\Phi}\left(x_{0}^{*}\right),-\frac{a+B x_{0}+e \alpha_{2}}{c+e^{T} x_{0}+b \alpha_{2}} \in \partial_{u} g_{\Phi}\left(x_{0}^{*}\right) \text {. }
$$

Another treatment of duality in fractional optimization, based on the notion of conjugation associated to a polarity introduced in [16], is provided in [17].

For the nonconvex duality theory based on $\Phi$-conjugation presented in this section a suitable generalized concept of Lagrangian was introduced in [9], encompassing the classical one of convex duality as well as a Lagrangian considered by Golstein [19] for fractional programming problems. A perturbational approach following a similar pattern as in convex optimization and a related notion of stability are provided in [10].

Some more details and further developments on nonconvex duality using $\Phi$ conjugate functions can be found in [13].

\section{References}

[1] Deumlich, R.; Elster, K.-H.: Generalized conjugate functions. Math. Operationsforsch. Statist. Ser. Optimization 8 (1977), no. 2, 151-179.

[2] Deumlich, R.; Elster, K.-H.: Duality theorems for nonconvex optimization problems. Math. Operations forsch. Statist. Ser. Optim. 3 (1978), no. 3, 335347.

[3] Deumlich, R.; Elster, K.-H.: On the theory of conjugate functions. Studies on mathematical programming (Papers, Third Conf. Math. Programming, Mátrafüred, 1975), pp. 19-45, Math. Methods Oper. Res., 1, Akad. Kiadó, Budapest, 1980.

[4] Deumlich, R.; Elster, K.-H.: Duality theorems and optimality conditions for nonconvex optimization problems. Math. Operations forsch. Statist. Ser. Optim. 11 (1980), no. 2, 181-219. 
[5] Deumlich, R.; Elster, K.-H.: $\Phi$-conjugation and nonconvex optimization. A survey. I. Math. Operationsforsch. Statist. Ser. Optim. 14 (1983), no. 1, 125149.

[6] Deumlich, R.; Elster, K.-H.: $\Phi$-conjugation and nonconvex optimization. A survey. II. Math. Operationsforsch. Statist. Ser. Optim. 15 (1984), no. 4, $499-515$.

[7] Deumlich, R.; Elster, K.-H.: Fractional programming in view of generalized conjugation. IX symposium on operations research. Part I. Sections 1-4 (Osnabrück, 1984), 3-16, Methods Oper. Res., 49, Athenäum/Hain/Hanstein, Königstein, 1985.

[8] Deumlich, R.; Elster, K.-H.: Contributions to duality theory of certain nonconvex optimization problems. Contributions to operations research (Oberwolfach, 1984), 10-40, Lecture Notes in Econom. and Math. Systems, 240, Springer, Berlin, 1985.

[9] Deumlich, R.; Elster, K.-H.: $\Phi$-conjugation and nonconvex optimization. III. A survey. Optimization 16 (1985), no. 6, 789-803.

[10] Deumlich, R.; Elster, K.-H.: On perturbations of certain nonconvex optimization problems. J. Optim. Theory Appl. 48 (1986), no. 1, 81-93.

[11] Deumlich, R.; Elster, K.-H.; Nehse, R.: Generalizations of conjugate functions. Survey of mathematical programming. Vol. 1. Proceedings of the Ninth International Mathematical Programming Symposium held in Budapest, August 23-27, 1976. Edited by András Prékopa. North-Holland Publishing Co., Amsterdam-Oxford-New York, 1979, pp. 193-204.

[12] Elster, K.-H.: On the theory of nonconvex optimization problems. Third Symposium on Operations Research (Univ. Mannheim, Mannheim, 1978), Section I, pp. 173-184, Operations Res. Verfahren, 31. Hain, Königstein/Ts., 1979.

[13] Elster, K.-H.: On duality results in nonconvex optimization. Functional analysis, optimization, and mathematical economics, 235-247, Oxford Univ. Press, New York, 1990. 
[14] Elster, K.-H.; Göpfert, A.: Conjugation concepts in optimization. XIV Symposium on Operations Research (Ulm, 1989), 53-65, Methods Oper. Res., 62, Hain, Frankfurt am Main, 1990.

[15] Elster, K.-H.; Wolf, A.: Comparison between several conjugation concepts. Optimal control (Oberwolfach, 1986), 79-93, Lecture Notes in Control and Inform. Sci., 95, Springer, Berlin, 1987.

[16] Elster, K.-H.; Wolf, A.: Recent results on generalized conjugate functions. Trends in mathematical optimization (Irsee, 1986), 67-78, Internat. Schriftenreihe Numer. Math., 84, Birkhäuser, Basel, 1988.

[17] Elster, K.-H.; Wolf, A.: Generalized convexity and fractional optimization. Generalized convexity and fractional programming with economic applications (Pisa, 1988), 219-231, Lecture Notes in Econom. and Math. Systems, 345, Springer, Berlin, 1990.

[18] Fenchel, W.: On conjugate convex functions. Canadian J. Math. 1, (1949). 73-77.

[19] Golstein,E . G.: Dualitätstheorie in der nichtlinearen Optirnierungi and ihre Anwendung. Akademie Verlag, Beriin, 1975.

[20] Moreau, J.-J.: Inf-convolution, sous-additivité, convexité des fonctions numériques. J. Math. Pures Appl. (9) 49 1970 109-154.

[21] Singer, I.: Abstract convex analysis. Canadian Mathematical Society Series of Monographs and Advanced Texts. A Wiley-Interscience Publication. John Wiley and Sons, Inc., New York, 1997. 